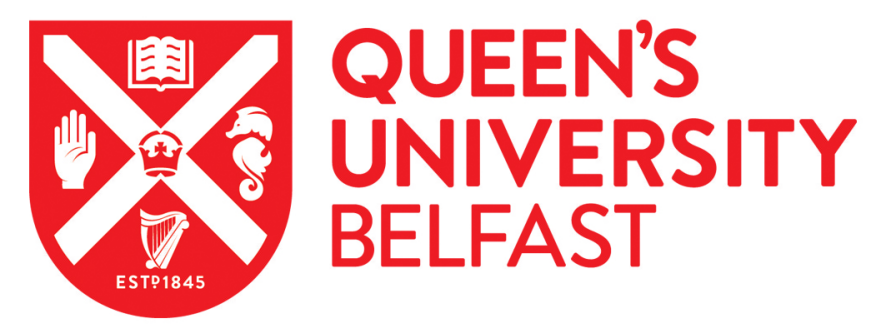

\title{
Increased rate of solvent diffusion in a prototypical supramolecular gel measured on the picosecond timescale
}

\author{
Seydel, T., Edkins, R. M., Jones, C. D., Foster, J. A., Bewley, R., Aguilar, J. A., \& Edkins, K. (2018). Increased \\ rate of solvent diffusion in a prototypical supramolecular gel measured on the picosecond timescale. Chemical \\ Communications, 54, 6340-6343. https://doi.org/10.1039/C8CC02962H
}

Published in:

Chemical Communications

Document Version:

Peer reviewed version

Queen's University Belfast - Research Portal:

Link to publication record in Queen's University Belfast Research Portal

Publisher rights

(c) 2018 Royal Society of Chemistry.

This work is made available online in accordance with the publisher's policies. Please refer to any applicable terms of use of the publisher.

\section{General rights}

Copyright for the publications made accessible via the Queen's University Belfast Research Portal is retained by the author(s) and / or other copyright owners and it is a condition of accessing these publications that users recognise and abide by the legal requirements associated with these rights.

Take down policy

The Research Portal is Queen's institutional repository that provides access to Queen's research output. Every effort has been made to ensure that content in the Research Portal does not infringe any person's rights, or applicable UK laws. If you discover content in the Research Portal that you believe breaches copyright or violates any law, please contact openaccess@qub.ac.uk. 


\section{Accepted Manuscript}

This article can be cited before page numbers have been issued, to do this please use: T. Seydel, R. M. Edkins, C. D. Jones, J. Foster, R. Bewley, J. A. A. Aguilar and K. Edkins, Chem. Commun., 2018, DOI:
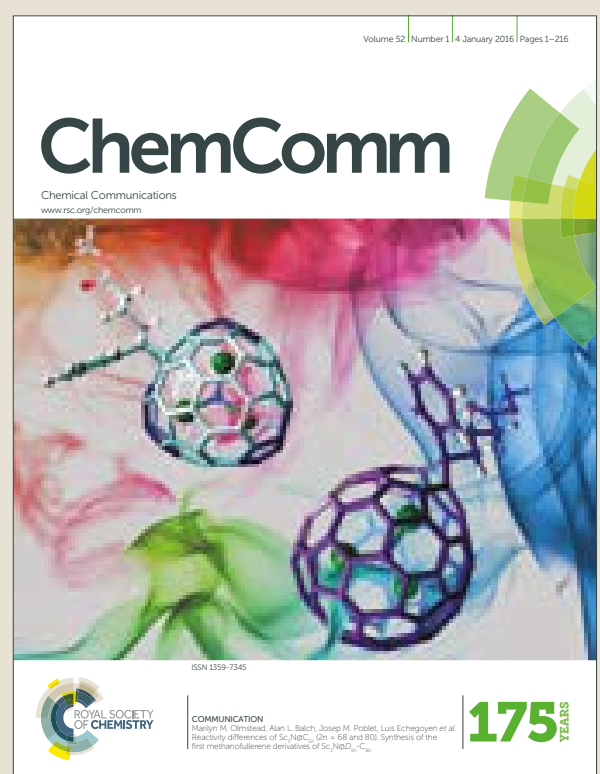

This is an Accepted Manuscript, which has been through the Royal Society of Chemistry peer review process and has been accepted for publication.

Accepted Manuscripts are published online shortly after acceptance, before technical editing, formatting and proof reading. Using this free service, authors can make their results available to the community, in citable form, before we publish the edited article. We will replace this Accepted Manuscript with the edited and formatted Advance Article as soon as it is available.

You can find more information about Accepted Manuscripts in the author guidelines.

Please note that technical editing may introduce minor changes to the text and/or graphics, which may alter content. The journal's standard Terms \& Conditions and the ethical guidelines, outlined in our author and reviewer resource centre, still apply. In no event shall the Royal Society of Chemistry be held responsible for any errors or omissions in this Accepted Manuscript or any consequences arising from the use of any information it contains. 


\section{Journal Name}

\section{COMUNICATION}

\section{Increased rate of solvent diffusion in a prototypical supramolecular gel measured on the picosecond timescale}

Received 00th January 20xx, Accepted 00th January 20xx

DOI: $10.1039 / \times 0 \times x 00000 x$

\section{Tilo Seydel, ${ }^{a}$ Robert M. Edkins, ${ }^{\mathrm{b}, \mathrm{c}}$ Christopher D Jones, ${ }^{\mathrm{d}}$ Jonathan A. Foster, ${ }^{\mathrm{e}}$ Robert Bewley, ${ }^{f}$ Juan} A. Aguilarg and Katharina Edkins ${ }^{\mathrm{d}^{*}}$

www.rsc.org/

Solvent diffusion in a prototypical supramolecular gel probed by quasi-elastic neutron scattering on the picosecond timescale is faster than that in the respective bulk solvent. This phenomenon is hypothesised to be due to disruption of the hydrogen bonding of the solvent by the large hydrophobic surface of the gel network.

The development of novel drug-delivery vehicles to safely transport drug compounds to the target tissue is of utmost importance to modern healthcare. ${ }^{1,2}$ Generating the correct release kinetics from the medicine reduces the risk of adverse drug reactions with severity ranging from mild discomfort to life-threatening, ${ }^{3}$ which also often lead to further costs for healthcare systems. We are interested in designing a new delivery vehicle for subcutaneous injection by utilizing supramolecular gels with tailored release kinetics. This emerging class of materials is based on small organic molecules that aggregate in 1D fibrils and fibers through intermolecular interactions such as hydrogen bonds, halogen bonds or $\pi$ stacking. ${ }^{4}$ The aggregation can be triggered by various physical and chemical stimuli, and the ease of changing their physicochemical characteristics by modification of the chemical structure makes them promising materials for drug-delivery. Urea-tape-forming gelators represent a large class of supramolecular gelators that can form hydrogels or organogels depending on the terminal substituents used. ${ }^{5-7}$ The gelator selected for this study (gelator 1, Figure 1a) has been reported to form a heat-set gel with a wide range of solvents. Most interesting for potential pharmaceutical applications is the

a. Institut Laue-Langevin, Boulevard des Martyrs, Grenoble Cedex 1, France

b. Chemistry Research Laboratory, University of Oxford, 12 Mansfield Road, Oxford, OX1 3TA, UK

c. Department of Pure and Applied Chemistry, Technology and Innovation Centre, University of Strathclyde, 99 George Street, Glasgow G1 1RD, UK

d. School of Pharmacy, Queen's University Belfast, 97 Lisburn Road, Belfast, BT9 $7 B L, U K$

e. Department of Chemistry, Sheffield University, Sheffield, S3 7HF, UK

f. ISIS Neutron Facility, Rutherford Appleton Laboratory, Didcot, OX11 OQX, UK

g. Chemistry Department, Durham University, South Road, Durham, DH1 3LE, UK

Electronic Supplementary Information (ESI) available: [details of any supplementary information available should be included here]. See DOI: 10.1039/x0xx00000x ability of gelator 1 to form hydrogels in the presence of small quantities of solubilizing, safe solvents such as ethanol $(0.175$ mole fraction, 7:3v/v water:ethanol). ${ }^{8}$

While the gelling behavior of supramolecular gels is routinely investigated and normally well described, the dynamics of these systems and especially diffusion kinetics are rarely published. ${ }^{9}$ The potential use of supramolecular gels for drug delivery, or in fact any tailored application, requires a detailed understanding of the complex molecular interactions and dynamics between the gelator, solvent and drug/solute molecules at the solid-liquid interface within the material. Before considering the multi-component system of a drugloaded gel, in this communication we report the diffusion of pure solvent within a 7:3 v/v water:ethanol gel of gelator 1 compared to that of the bulk solvent.

Quasi-elastic neutron scattering (QENS) measurements probe dynamics on the sub-nanosecond timescale and nanometer length-scale, making it highly suitable for studying solvent confined within the nanoporous scaffold of the gels. The technique measures the energy transfer from the incident monochromated neutron beam onto the sample due to scattering from diffusing molecules; thus, the faster the molecules diffuse in the sample, the broader the energy distribution of the scattered beam. QENS of gel samples of gelator 1 was measured at two concentrations $(0.3$ and $0.5 \%$ $w / v)$ along with the bulk solvent for comparison. Samples were measured at four different, pharmaceutically relevant temperatures $(275,285,295$ and $305 \mathrm{~K}$, see ESI for experimental section). The scattering data clearly show a broader peak for the gel samples at both concentrations than for the bulk solvent. Thus, this model-free comparison of the linewidths from the different samples indicates that the solvent diffuses faster in the gel samples (Figure $1 b$ ). The dependence of the peak width on the square of the momentum transfer $\left(q^{2}\right)$ allows us to associate the diffusion with a length scale and, thus, to determine a diffusion model. A linear dependence of the two values would indicate the simplest diffusion model, namely Brownian motion. 

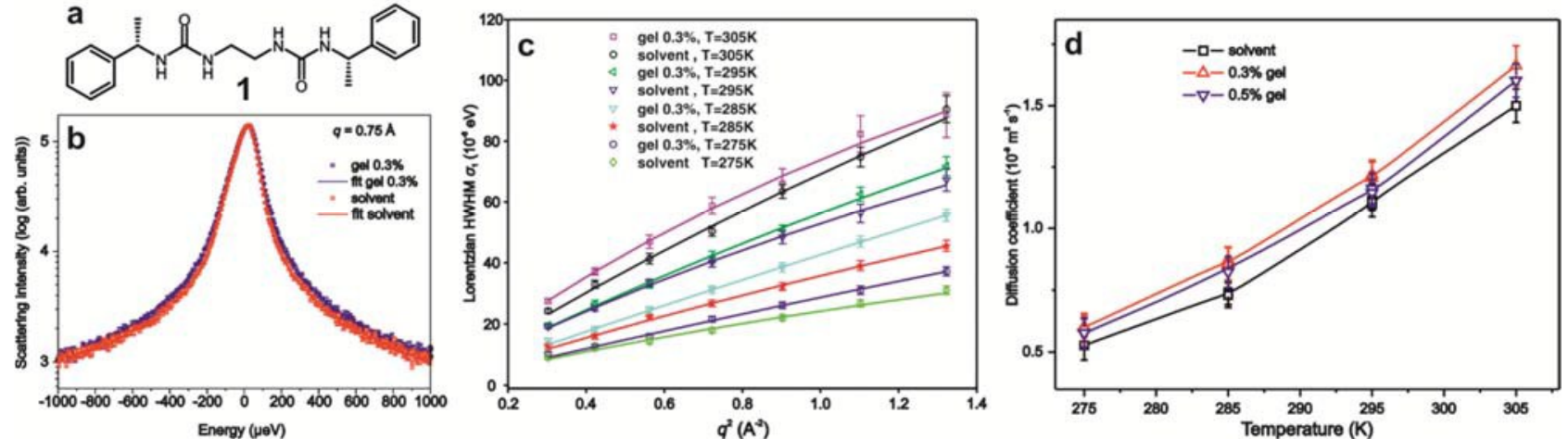

Figure 1. Picosecond diffusion measurements by QENS of solvent in gels formed from gelator 1: a) chemical structure of gelator 1, b) example spectra of bulk solvent and $0.3 \% \mathrm{w} / \mathrm{v}$ gel and their respective fits for an incident neutron energy of $3.4 \mathrm{meV}$, c) plot of half-width-at-half-maximum (HWHM) of the fitted Lorentzian $\sigma_{1} v s . q^{2}$ showing clear jump-diffusion behavior, d) fitted diffusion coefficients vs. temperature of bulk solvent, $0.3 \%$ and $0.5 \% w / v$ gels over the probed temperature range of the experiment. All error bars display $95 \%$ confidence.

In this model, the dynamics of the solvent are described by its self-diffusion coefficient $D$ only. In the presence of stronger intermolecular interactions, e.g. hydrogen bonds, the diffusion is hampered by the need to overcome an activation energy, resulting in a residence time $\tau$ between diffusion steps (jumpdiffusion model). This results in a non-linear dependence of the peak width on scattering angle, which is clearly visible in our data (Figure 1c).

For a quantitative interpretation of the QENS data, the jump-diffusion model ${ }^{10}$

$$
\sigma_{1}(q)=\frac{D q^{2}}{1+D q^{2} \tau}
$$

was fitted over the $q$-range measured, which allows for the extraction of values for $D$ and $\tau$ (Figure $1 \mathrm{~d}$, Table 1 and S1). For particulars of the fit, please refer to ESI. In line with the modelfree approach, all $D$-values of the gel samples at all temperatures are larger than those of the bulk solvent (by 10 $18 \%$ for the $0.3 \% w / v$ gel and $4-12 \%$ for the $0.5 \% w / v$ gel). This trend was observed for multiple, individually prepared samples over the whole temperature range probed, and for measurements conducted on two independent neutron scattering instruments. The result is thus reproducible and surprising in light of previous studies investigating solvent diffusion in soft materials and confinement. The only other QENS study of a low-molecular-weight gelator reports a slowing down of solvent diffusion due to strong interactions between the solvent molecules and the solvophilic gel fiber surface. ${ }^{11,12}$ Similarly, other QENS studies on polymeric hydrogels, 13,14 biopolymers ${ }^{15}$ and polysaccharides ${ }^{16}$ report a slowing down of the solvent in the gels. In instances in which the solvent is confined in pores comparable to those in the gel network, similar slowing-down behavior is found; a common system to study solvent diffusion is nanoporous silica typically measured or simulated on a sub-nanosecond timescale. ${ }^{17,} 18$ Highly hydrogen-bonded solvent, e.g. water, diffuses more slowly within these pores than in the bulk solvent. ${ }^{19}$ This behavior, which could be replicated by simulations, has been rationalized as being due to a static water layer above the pore surface. ${ }^{20} \mathrm{In}$ addition, the hydrophilicity or hydrophobicity of
Table 1. Representative fit results for the diffusion coefficients $D$ and residence times $\tau$ for various samples and temperatures as measured by QENS on the instrument LET (ISIS neutron source, UK) and fitted using a jump diffusion model.

\begin{tabular}{llclll} 
Sample & $T[\mathrm{~K}]$ & $D\left[10^{-9} \mathrm{~m}^{2} \mathrm{~s}^{-1}\right]$ & $2 \sigma$ on $D$ & $\tau$ [ps] & $2 \sigma$ on $\tau$ \\
\hline Solvent & 275 & 0.527 & 0.059 & 3.826 & 1.093 \\
Gel 0.3\% & 275 & 0.604 & 0.043 & 3.026 & 0.597 \\
Gel 0.5\% & 275 & 0.576 & 0.061 & 3.512 & 0.943 \\
\hline Solvent & 285 & 0.741 & 0.049 & 2.307 & 0.453 \\
Gel 0.3\% & 285 & 0.872 & 0.050 & 2.078 & 0.334 \\
Gel 0.5\% & 285 & 0.824 & 0.062 & 2.268 & 0.470 \\
\hline Solvent & 295 & 1.098 & 0.025 & 1.548 & 0.105 \\
Gel 0.3\% & 295 & 1.220 & 0.059 & 1.594 & 0.204 \\
Gel 0.5\% & 295 & 1.163 & 0.064 & 1.726 & 0.243 \\
\hline Solvent & 305 & 1.500 & 0.068 & 1.311 & 0.155 \\
Gel 0.3\% & 305 & 1.663 & 0.080 & 1.314 & 0.151 \\
Gel 0.5\% & 305 & 1.602 & 0.068 & 1.456 & 0.139 \\
\hline
\end{tabular}

the pores' surface does not play a major role in modifying water diffusion. ${ }^{21}$ Diffusion of water through the ultra-hydrophobic channels of carbon nanotubes, on the other hand, is quicker than in bulk solvent. ${ }^{22-25}$ Comparable to the hydrophobic inner surfaces of carbon nanotubes, the surface of fibers formed by gelator $\mathbf{1}$ is decorated with phenyl rings, giving these interfaces a similar hydrophobicity. We hypothesize that the fiber surfaces can thus interrupt the hydrogen bonding within the solvent and therefore lower the viscosity in the direct vicinity of the fibers. The result of this study opens up an experimental space to tune the diffusion of the liquid phase over the gel fiber network depending on its surface chemistry, which will be important for tailored drug delivery, where the diffusion coefficient of the liquid phase is expected to relate to drug release out of the application form. We also anticipate that our result has impact on other gel applications, such as selective crystallization, catalysis and sensing, in which solute molecules strongly interact with the fiber surface and where diffusion rate will determine the efficacy or selectivity.

In addition to the fiber-surface chemistry, the importance of the extent of fiber surface area in contact with the liquid phase on the solvent diffusion was experimentally validated in two ways. 

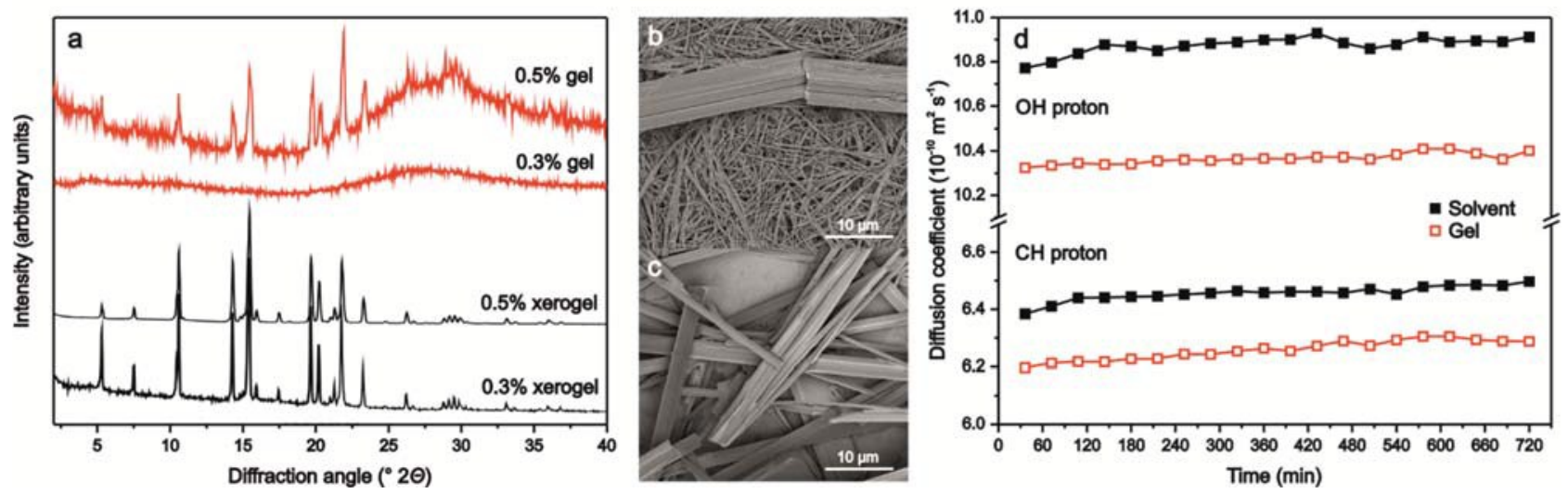

Figure 2 Material characterization of the gels: a) powder X-ray diffractograms of the gels (top) and the respective xerogels (bottom), SEM micrographs of b) $0.3 \% \mathrm{w} / \mathrm{v}$ and c) $0.5 \%$ $w / v$ xerogels, and d) diffusion coefficients of the solvent entrapped in the $0.3 \% w / v$ gel and bulk solvent as probed by ${ }^{1} \mathrm{H}$ DOSY NMR spectroscopy. Measurements were made over 12 hours to avoid artefacts caused by fluctuations in the magnetic field.

Firstly, when samples of $0.3 \% w / v$ gelator concentration were prepared without gelformation (precipitation observed after opening the sample container), the QENS data showed no difference in diffusion behavior to the bulk solvent. Thus, without the substantial increase in surface area due to gelformation, the gelator material has no measurable influence on solvent diffusion.

Secondly, samples using $0.3 \% w / v$ and $0.5 \% w / v$ gelator show an unexpected difference in diffusion coefficient. Even though both materials show a higher solvent diffusion coefficient compared to bulk solvent, the lower-concentration gel shows the quickest diffusion. It is hypothesized that the gel network in the two samples have distinct differences, with a larger surface area exposed to solvent in the $0.3 \% \mathrm{w} / \mathrm{v}$ gel. Oscillatory shear rheometry of both samples shows, besides the characteristic gel behavior, a continuous rise in the viscous modulus $G^{\prime \prime}$ on approaching yield stress, suggesting that a fraction of the gelator is not incorporated into the fibrous gel network but rather crystallizes in the sample (Figure S4). This phenomenon, known as weak strain overshoot, occurs due to competition between the formation and destruction of network junctions under stress, and is often observed when microcrystals and other discrete particles are suspended within a gel. ${ }^{26}$ Analysis by powder X-ray diffraction shows the mainly amorphous nature of both samples, but while no diffraction peaks are present in the $0.3 \% \mathrm{w} / \mathrm{v}$ sample, the more concentrated gel shows clear signs of crystallinity in the gel state. After drying, both samples show the highly crystalline pattern of the xero-gel (Figure 2a). ${ }^{7}$ Furthermore, scanning electron micrographs show the sub-micron fibrous structure of the $0.3 \% \mathrm{w} / \mathrm{v}$ gel with only a low fraction of larger particles (Figure $2 \mathrm{~b}$, Figure S5). At the higher concentration of $0.5 \% \mathrm{w} / \mathrm{v}$, the fibrous structure is almost completely replaced by larger particles (Figure 2c), leading to a much-reduced surface area. Even though electron microscopy is not necessarily showing the native structure of the gel phase due to changes during sample preparation, 27, 28 in our case this method corroborates our hypothesis. Whilst the smaller surface area leads to a reduced effect on the diffusion coefficient $D$ when compared to the lower concentrated gel, it is still sufficient to increase the solvent diffusion coefficient relative to the bulk. Clearly, the presence of the sub-micron gel fibers with their large surface area as observed in the $0.3 \% \mathrm{w} / \mathrm{v}$ gel is critical for even higher diffusion coefficients.

In addition to the picosecond timescale measurements, we probed solvent diffusion by ${ }^{1} \mathrm{H}$ diffusion-ordered spectroscopy (DOSY) NMR on the longer timescale of typically tens to hundreds of milliseconds (Figure 2d, Figure S6). This method has been used as a standard technique to investigate supramolecular gels, partly because this laboratory-based technique is readily available. Tritt-Goc and co-workers have reported a large volume of work in this field, and show for the investigated sugar-based gelators that the solvent diffusion slows down in the gels compared to the bulk in line with the QENS measurements of solvophilic gels. ${ }^{29-32}$ Having observed an opposite effect by QENS in the current study, we recorded DOSY spectra of gels at both gelator concentrations. Both types of proton signals $(\mathrm{CH}$ and $\mathrm{OH})$ reveal a distinctly slower diffusion of the solvent in the gel samples than in the bulk, in good agreement with previous NMR-based studies. ${ }^{29-32}$ Even though seemingly counter to the QENS results, the two measurements are not inconsistent. On the short diffusion timescale probed by QENS, the solvent molecules diffuse over a distance of nanometers and it is highly unlikely that they impact on another fiber or fiber junction. Diffusion is thus only influenced by the surface of a single fiber and the local viscosity near the surface. On the longer timescales of a DOSY measurement, the solvent molecules diffuse micrometers and therefore much larger distances than the typical dimensions of confinement, which makes it more likely that they impact with other gel fibers and thus deviate from the diffusion model of a free random walk. The apparent diffusion vector of a solvent molecule in the gel probed on the timescale of DOSY measurements can thus be shorter, even though the total distance covered is comparable to that extrapolated from the diffusion coefficients measured by QENS. This phenomenon has been shown for solvent diffusion in confinement ${ }^{33}$ and is used to probe pore sizes in biological materials such as the white matter of the brain, 34,35 
or in functional materials such as mesoporous silica. ${ }^{36}$ It is thus not sufficient to investigate diffusion behavior of supramolecular gels by QENS or NMR spectroscopy alone, as only a partial description can be gained with a single technique.

In conclusion, quasi-elastic neutron scattering has been used to probe the diffusion behavior of solvent $(7: 3 \mathrm{v} / \mathrm{v}$ ethanol:water) in a supramolecular gel based on a prototypical small molecule gelator and has shown that the solvent diffuses faster in the gel network compared to the bulk solvent on the picosecond timescale and nanometer length scale. This is attributed to the large surface area of the hydrophobic fibers in the gel network breaking the hydrogen bonding of the solvent and thus lowering the viscosity in the direct vicinity of the fibers. This effect exists to a lower extent in higher concentration gels, which contain a larger amount of crystalline aggregates, thus lowering the surface area presented to the solvent molecules. Diffusion measurements on the longer timescale probed by NMR spectroscopy (milliseconds vs. picoseconds) show the reversal of the effect with solvent diffusion in the gels being slower than that in bulk solvent. This can be explained by the probability of impact on gel fibers, which renders the solvent molecule a non-free random walker and leads to a slower apparent diffusion coefficient. The use of only one experimental method to probe the solvent diffusion is thus not sufficient and can lead to underestimation of the diffusion rate over the gel fibers. Building on the results of this study, we can now begin to engineer the fiber surface according to the required diffusion characteristics of the liquid phase.

\section{Acknowledgements}

We would like to thank Prof. Jon W. Steed for support, discussion and supply of gelator 1 . The Institut Laue-Langevin and ISIS neutron facilities are thanked for the allocation of neutron beamtime (experiments 7-04-80, RB1410058 and RB1510163; these data are permanently curated by the ILL and ISIS, respectively, and accessible by these reference numbers). R.M.E. thanks the Royal Commission for the Exhibition of 1851 , and J.A.F. thanks the Ramsay Memorial Trust for a research fellowship.

\section{Conflicts of interest}

The authors declare no conflicts of interest.

\section{Notes and references}

1. W. Tan, H. Wang, Y. Chen, X. Zhang, H. Zhu, C. Yang, R. Yang and C. Liu, Trends Biotechnol., 2011, 29, 634-640.

2. R. F. Donnelly, T. R. R. Singh and A. D. Woolfson, Drug deliv., 2010, 17, 187-207.

3. I. R. Edwards and J. K. Aronson, The Lancet, 2000, 356, 12551259.

4. M. George and R. G. Weiss, Acc. Chem. Res., 2006, 39, 489497.

5. J. A. Foster, M. O. M. Piepenbrock, G. O. Lloyd, N. Clarke, J. A. K. Howard and J. W. Steed, Nature Chem., 2010, 2, 10371043.
6. M.-O. M. Piepenbrock, G. O. Lloyd, N. Clarke and J. W. Steed, Chem. Commun., 2008, 0, 2644-2646.

7. G. O. Lloyd, M.-O. M. Piepenbrock, J. A. Foster, N. Clarke and J. W. Steed, Soft Matter, 2012, 8, 204-216.

8. Food and Drug Administration: Guideline Q3C Impurities: Residual Solvent; Food and Drug Administration: Generaly Recognised as Safe (GRAS) guidelines

9. E. R. Draper and D. J. Adams, Chem, 2017, 3, 390-410.

10. J. Qvist, H. Schober and B. Halle, J. Chem. Phys., 2011, 134, 031101.

11. S. Spagnoli, I. Morfin, M. A. Gonzalez, P. Çarçabal and M. Plazanet, Langmuir, 2015, 31, 2554-2560.

12. I. Morfin, S. Spagnoli, C. Rambaud, S. Longeville and M. Plazanet, Philosop. Mag., 2016, 96, 809-815.

13. G. Paradossi, F. Cavalieri, E. Chiessi and M. T. F. Telling, J. Phys. Chem. B, 2003, 107, 8363-8371.

14. G. Paradossi, I. Finelli, F. Natali, M. T. F. Telling and E. Chiessi, Polymers, 2011, 3, 1805-1832.

15. A. Deriu, F. Cavatorta, D. Cabrini, C. J. Carlile and H. D. Middendorf, Europhys. Lett., 1993, 24, 351-357.

16. F. Cavalieri, E. Chiessi, I. Finelli, F. Natali, G. Paradossi and M. F. Telling, Macromol. Biosci., 2006, 6, 579-589.

17. D. G. Lev, K. E. Gubbins, R. Radhakrishnan and M. SliwinskaBartkowiak, Rep. Prog. Phys., 1999, 62, 1573-1659.

18. J. Teixeira, J. M. Zanotti, M. C. Bellissent-Funel and S. H. Chen, Physica B, 1997, 234-236, 370-374.

19. I. M. Briman, D. Rébiscoul, O. Diat, J.-M. Zanotti, P. Jollivet, P. Barboux and S. Gin, J. Phys. Chem. C, 2012, 116, 7021-7028.

20. I. C. Bourg and C. I. Steefel, J. Phys. Chem. C, 2012, 116, 11556-11564.

21. D. Yu, D. Page, J. Li, Y. Mastau, M. Koza and G. Kearley, J. Phys. Soc. Jpn., 2013, 82, SA011.

22. G. Hummer, J. C. Rasaiah and J. P. Noworyta, Nature, 2001, 414, 188-190.

23. J. K. Holt, H. G. Park, Y. Wang, M. Stadermann, A. B. Artyukhin, C. P. Grigoropoulos, A. Noy and O. Bakajin, Science, 2006, 312, 1034-1037.

24. D. Mattia and F. Calabrò, Microfluid. Nanofluid., 2012, 13, 125-130.

25. S. Joseph and N. R. Aluru, Nano Lett., 2008, 8, 452-458.

26. K. Hyun, M. Wilhelm, C. O. Klein, K. S. Cho, J. G. Nam, K. H. Ahn, S. J. Lee, R. H. Ewoldt and G. H. McKinley, Prog. Polym. Sci., 2011, 36, 1697-1753.

27. D. J. Adams, Gels, 2018, 4, 32.

28. L. L. E. Mears, E. R. Draper, A. M. Castilla, H. Su, Zhuola, B. Dietrich, M. C. Nolan, G. N. Smith, J. Doutch, S. Rogers, R. Akhtar, H. Cui and D. J. Adams, Biomacromolecules, 2017, 18, 3531-3540.

29. M. Bielejewski, J. Kowalczuk, J. Kaszynska, A. Lapinski, R. Luboradzki, O. Demchuk and J. Tritt-Goc, Soft Matter, 2013, 9, 7501-7514

30. J. Kowalczuk, M. Bielejewski, A. Lapinski, R. Luboradzki and J. Tritt-Goc, J. Phys. Chem. B, 2014, 118, 4005-4015.

31. J. Tritt-Goc, A. Rachocki and M. Bielejewski, Soft Matter, 2014, 10, 7810-7818

32. J. Kowalczuk, A. Rachocki, M. Bielejewski and J. Tritt-Goc, J. Colloid Interf. Sci., 2016, 472, 60-68.

33. P. P. Mitra, P. N. Sen, L. M. Schwartz and P. Le Doussal, Phys. Rev. Lett., 1992, 68, 3555-3558.

34. A. F. Frøhlich, S. N. Jespersen, L. Østergaard and V. G. Kiselev, J. Magn. Res., 2008, 194, 128-135.

35. E. Fieremans, Y. De Deene, S. Delputte, M. S. Özdemir, Y. D'Asseler, J. Vlassenbroeck, K. Deblaere, E. Achten and I. Lemahieu, J. Magn. Res., 2008, 190, 189-199.

36. T. J. Rottreau, C. M. A. Parlett, A. F. Lee and R. Evans, J. Phys. Chem. C, 2017, 121, 16250-16256. 